\title{
Behavior of magnetic impurities in gapless Fermi systems
}

\author{
Kevin Ingersentt \\ Department of Physics, University of Florida, P. O. Box 118440, Gainesville, Florida 32611-8440, USA
}

(Revised version: 13 May 1996)

\begin{abstract}
In a number of systems, including certain semiconductors and unconventional superconductors, the effective density of states varies near the Fermi energy like $\left|E-E_{F}\right|^{r}$. The behavior of dilute magnetic impurities in such systems is studied using a non-perturbative renormalization-group approach. Close to particle-hole symmetry, the Kondo effect is suppressed for the cases of greatest relevance $(r=1$ and 2). Away from this symmetry, any quenching of the impurity moment is accompanied by a low-temperature decrease in the impurity resistivity, rather than the increase found in metals.
\end{abstract}

75.20.Hr, 74.70.Tx, 72.15.Qm

In an interesting class of "gapless" Fermi systems, the density of states $\rho(\epsilon)$ vanishes right at the Fermi energy $E_{F}$, but not at any other energy near $\epsilon \equiv E-E_{F}=0$. For instance, the quasiparticle density of states in an unconventional superconductor can vary like $|\epsilon|$ or $|\epsilon|^{2}$ near line or point nodes in the gap [1]. Heavy-fermion and cuprate superconductors are candidates for this behavior. The valence and conduction bands of certain semiconductors - including $\mathrm{Pb}_{1-x} \mathrm{Sn}_{x} \mathrm{Te}$ at a critical composition [2], and PbTe-SnTe heterojunctions [3] — touch in such a way that, for small $|\epsilon|, \rho(\epsilon)$ is proportional to $|\epsilon|^{d-1}$ in $d$ spatial dimensions. Electrons in a strong magnetic field (at least in the absence of disorder) [4] and exotic phases of the Hubbard model [5] are also predicted to exhibit a linear pseudo-gap in two dimensions.

Antiferromagnetic coupling between magnetic impurities and a metallic conduction band leads to a lowtemperature increase in the resistivity and a reduction in the Curie term in the susceptibility. This Kondo effect depends on the existence of electronic excitations down to zero energy, and thus cannot be fully realized in systems with a finite energy gap. Gapless systems with a density of states varying like $|\epsilon|^{r}$ constitute a marginal case, first studied in Ref. [6]. Poor-man's scaling for the spin- $\frac{1}{2}$ (impurity degeneracy $N=2$ ) Kondo model, and a large- $N$ treatment restricted to $r<\frac{1}{2}$ (but recently extended [7] to include $r=1$ ), both showed that a Kondo effect takes place only if the electron-impurity exchange $J$ exceeds a critical value, $J_{c} \propto r$; otherwise, the impurity decouples from the band. A large- $N$ treatment of magnetic impurities in gapless superconductors [8] yielded similar results, except that for $r \leq 1$ or $N=2$, any finite impurity concentration was found to drive $J_{c}$ to zero. Recently, however, third-order scaling was applied to the $n$-channel Kondo model to show that, at least for $n \gg 1$, no Kondo effect can occur if $r>1 /(2 n)$ 9].

This paper reports the results of a nonperturbative renormalization-group (RG) study of a spin- $\frac{1}{2}$ impurity interacting with an electronic band in which $\rho(\epsilon)$ takes one of several functional forms, each varying like $|\epsilon|^{r}$ near $\epsilon=0$. A stability analysis of the RG fixed points and numerical calculations of impurity thermodynamic prop- erties are presented. Particle-hole asymmetry is identified as a key factor in determining the low-temperature physics. At small asymmetry, the critical coupling $J_{c}$ above which the impurity moment is screened becomes so large for all $r>\frac{1}{2}$ that the Kondo effect is suppressed. Larger asymmetries produce a low-temperature decrease in the impurity resistivity, rather than the monotonic increase which characterizes the metallic Kondo effect.

After the completion of this work, the author became aware of a similar study, limited to particle-holesymmetric systems and a pure power-law density of states with $0<r \leq 1$ [10]. The present paper confirms the conclusions of Ref. [10] for this special case, but shows that the generic behavior of gapless systems is quite different.

The Kondo Hamiltonian describing impurity potential and exchange scattering of band electrons can be written

$$
\begin{aligned}
H \equiv & H_{\text {band }}+H_{\mathrm{imp}}=D \sum_{\sigma} \int d \varepsilon \varepsilon c_{\varepsilon \sigma}^{\dagger} c_{\varepsilon \sigma} \\
& +V \sum_{\sigma} f_{0 \sigma}^{\dagger} f_{0 \sigma}+J \sum_{\sigma, \sigma^{\prime}} f_{0 \sigma}^{\dagger} \frac{1}{2} \sigma_{\sigma \sigma^{\prime}} f_{0 \sigma^{\prime}} \cdot \mathbf{S} .
\end{aligned}
$$

Here $\varepsilon=\left(E-E_{F}\right) / D$ is a reduced kinetic energy, measured from the Fermi level in an isotropic band of width $2 D ; J>0$ represents antiferromagnetic exchange; $c_{\varepsilon \sigma}$ annihilates a spin- $\sigma$ electron in an $s$-wave state of reduced energy $\varepsilon$; and $f_{0 \sigma}=\int d \varepsilon \sqrt{D \rho(\varepsilon D)} c_{\varepsilon \sigma}$ is the combination of $c_{\varepsilon \sigma}$ 's that destroys an electron at the impurity site. The fermionic operators are normalized such that $\left\{c_{\varepsilon \sigma}^{\dagger}, c_{\varepsilon^{\prime} \sigma^{\prime}}\right\}=\delta\left(\varepsilon-\varepsilon^{\prime}\right) \delta_{\sigma, \sigma^{\prime}}$ and $\left\{f_{0 \sigma}^{\dagger}, f_{0 \sigma^{\prime}}\right\}=\delta_{\sigma, \sigma^{\prime}}$ [11].

Gapless systems can be modeled by a density of states

$$
\rho(\epsilon) \equiv \rho(\varepsilon D)= \begin{cases}\rho_{0}|\varepsilon|^{r} & \text { if }|\varepsilon| \leq 1 \\ 0 & \text { otherwise }\end{cases}
$$

with $r>0$. This form is oversimplified, but it will turn out that for practical purposes the results are little changed if one introduces band asymmetry, limits the power-law variation to the vicinity of $E_{F}$, or allows $\rho(0)$ to be small but nonzero. Standard results [12 should (and will) be recovered in the metallic limit, $r=0$.

Equation (17) can be treated nonperturbatively using a generalization of Wilson's numerical RG method 12] 
to an arbitrary density of states. The Hamiltonian is written $H=\lim _{M \rightarrow \infty} D \Lambda^{-M / 2} \tilde{H}_{M}$, where

$$
\begin{aligned}
\tilde{H}_{M}= & \Lambda^{1 / 2} \tilde{H}_{M-1}+\sum_{\sigma}\left[\varepsilon_{M} f_{M \sigma}^{\dagger} f_{M \sigma}\right. \\
& \left.+\xi_{M-1}\left(f_{M \sigma}^{\dagger} f_{M-1, \sigma}+\text { h.c. }\right)\right]
\end{aligned}
$$

for $M \geq 0$. Here $\tilde{H}_{-1}=H_{\text {imp }} /\left(\Lambda^{1 / 2} D\right)$ and $\xi_{-1}=0$; $\Lambda>1$ parameterizes the logarithmic discretization of the conduction band [12]; and $f_{M}$ annihilates an electron in a state, centered on the impurity site, that becomes progressively more delocalized as $M$ increases. Numerical solutions of successive $\tilde{H}_{M}$ 's capture the key physics at a sequence of temperatures $T \approx D \Lambda^{-M / 2}$ 12, 13].

All dependence on $\rho(\epsilon)$ is contained in $\xi_{M}$ and $\varepsilon_{M}$, which are given by a set of Lanczos recursion relations. Quite generally, if $\rho(\varepsilon)=\rho(-\varepsilon)$, then $\varepsilon_{M}=0$ for all $M$. For the density of states in Eq. (2), one finds [14] that

$$
\xi_{M} \stackrel{M \rightarrow \infty}{\longrightarrow} \frac{1+r}{2+r} \frac{1-\Lambda^{-(2+r)}}{1-\Lambda^{-(1+r)}} \Lambda^{-1 / 2} \Lambda^{-(M \bmod 2) r / 2} .
$$

Preliminary analysis: Even before one resorts to heavy computation, study of the limits $J=0$ and $\infty$ provides valuable insight concerning the density of states given by Eq. (2): (1) For all $r>0$, there is a weak-coupling regime in which the Kondo interaction is irrelevant [6]. (2) At particle-hole symmetry, a Kondo effect (i.e., scaling to infinite $J$ ) is ruled out for all $r>\frac{1}{2}$ [9.10]. (3) If $V \neq 0$ in Eq. (1), there is a stable strong-coupling limit in which the impurity is completely screened, but the impurity resistivity is zero rather than taking its maximum possible value, as it does in metals. (4) Since each RG trajectory must flow from an unstable fixed point to a stable one, the intermediate-coupling region is also constrained. If the limit $J=\infty$ is stable, at least one (unstable) fixed point must lie at some $0<J_{c}<\infty$, whereas instability about $J=\infty$ is consistent with uninterrupted RG flow from strong to weak coupling. The derivation of these results, outlined in the next four paragraphs, follows Ref. [12].

For $J=0$, different electron spins decouple. Each spin is described by an effective Hamiltonian $\tilde{H}_{M}^{(0)}(V)$, where

$$
\begin{aligned}
\tilde{H}_{M}^{(L)}(V)= & \Lambda^{M / 2}\left[(V / D) f_{0}^{\dagger} f_{0}\right. \\
& \left.+\sum_{m=L}^{M-1} \Lambda^{-m / 2} \xi_{m}\left(f_{m}^{\dagger} f_{m+1}+\text { h.c. }\right)\right]
\end{aligned}
$$

For $M \gg 1$, it is found numerically that the low-lying eigenvalues of $\tilde{H}_{M}^{(0)}(V)$ are independent of $M$ and $V$, while the $f$ 's have simple expansions in terms of the exact eigenoperators $g_{0}, \ldots, g_{M}: f_{0}=\Lambda^{-(1+r) M / 4} \sum_{j} \alpha_{j} g_{j}$, where $\alpha_{j}$ is independent of $M$; all other $f_{m}$ 's decay with increasing $M$ at least as fast as $f_{0}$ decays. The impurity susceptibility is Curie-like, $T \chi_{\mathrm{imp}}=\frac{1}{4}$, and the entropy is
$S_{\mathrm{imp}}=\ln 2$ [13. For $r>0$, electrons at the Fermi energy scatter from the impurity with a phase shift $\delta(\epsilon=0)=0$.

Any deviation of $\tilde{H}_{M}$ from $\tilde{H}_{M}^{(0)}(V)$ must be a combination of $f^{\dagger}$ 's and $f$ 's which respects all symmetries of Eqs. (11) and (2), multiplied by the same factor of $\Lambda^{M / 2}$ as enters Eq. (5). Using the above expansion of the $f$ 's and the relation $T \approx D \Lambda^{-M / 2}$, one can classify the relevance of any such operator 12]. For instance, the exchange and potential-scattering operators, $\mathcal{O}_{J}=\Lambda^{M / 2} f_{0 \sigma}^{\dagger} \frac{1}{2} \boldsymbol{\sigma}_{\sigma \sigma^{\prime}} f_{0 \sigma^{\prime}} \cdot \mathbf{S}$ and $\mathcal{O}_{V}=\Lambda^{M / 2} f_{0 \sigma}^{\dagger} f_{0 \sigma}$, both vary like $T^{r}$, a temperature dependence which can be absorbed into effective couplings $J_{\text {eff }}(T)=J \cdot(T / D)^{r}$ and $V_{\text {eff }}(T)=V \cdot(T / D)^{r}$. Any other allowed operator has an algebraically greater temperature exponent. Thus, for all $r>0, \mathcal{O}_{J}$ and $\mathcal{O}_{V}$ are the leading irrelevant operators about a stable $J=0$ fixed point. In the metallic case $(r=0)$, by contrast, $J_{\text {eff }}$ grows logarithmically as $T$ decreases, so the fixed point is marginally unstable.

In the limit $J=\infty$ (with $V$ finite), an $f_{0}$ electron is locked into a spin singlet with the impurity. Hopping to or from $f_{0}$ states is ruled out, so different electron spins again decouple. The effective Hamiltonian is $\tilde{H}_{M}^{(1)}(0)$ given by Eq. (5). Now $f_{m} \propto \Lambda^{-\left(1-r_{1}\right) M / 4}$ for $m$ odd and $f_{m} \propto \Lambda^{-\left(3-r_{3}\right) M / 4}$ for $m$ even, where $r_{n}=\min (r, n)$. One finds that $T \chi_{\mathrm{imp}}=r_{1} / 8, S_{\mathrm{imp}}=2 r_{1} \ln 2$, and $\delta(0)=\left(1-r_{1}\right) \pi / 2$, indicating that even at $J=\infty$ the impurity degree of freedom is not completely quenched, but instead is partially transferred to the band [10].

The stability of this fixed point hinges on whether or not the problem is particle-hole symmetric. If $V=0$ in Eq. (1), the most relevant perturbation about $\tilde{H}_{M}^{(1)}$ is $\Lambda^{M / 2}\left(f_{1}^{\dagger} f_{1}-\frac{1}{2}\right)^{2} \propto T^{1-2 r_{1}}$, in which case the fixed point is stable for $r<\frac{1}{2}$, but is destabilized for all $r>\frac{1}{2}$. Setting $V \neq 0$ admits an additional operator, $\Lambda^{M / 2} f_{1 \sigma}^{\dagger} f_{1 \sigma} \propto T^{-r_{1}}$. For $r>0$, this freezes the $f_{1}$ states as $T \rightarrow 0$ and drives the system to a new $J=\infty$ fixed point, described by $\tilde{H}_{M}^{(2)}(0)$. Due to the "odd-even" character of the Hamiltonians $\tilde{H}_{M}^{(L)}, \tilde{H}_{M}^{(2)}(0)$ has the same low-energy spectrum and stability properties as the $J=0$ Hamiltonian $\tilde{H}_{M}^{(0)}(0)$. At this fixed point, $T \chi_{\mathrm{imp}}=0$, $S_{\mathrm{imp}}=0$, and $\delta(0)=\pi$; the impurity contribution to the resistivity, $\varrho \propto \sin ^{2} \delta(0)$, vanishes for $r>0$.

Numerical results: The RG picture has been completed by solving the Hamiltonians $\tilde{H}_{M}$ in Eq. (3) for arbitrary $J$. The entropy, heat capacity and magnetic susceptibility have been computed by adapting methods developed for the metallic Kondo problem 12,15. (Space permits only the susceptibility to be plotted.) The main sources of error are the discretization of the conduction band and the truncation of the basis of $\tilde{H}_{M}$. In this work 800-2000 states were retained, sufficiently many that truncation errors are negligible (smaller than the symbols in Figs. 1 3). All data shown were obtained using a discretization parameter $\Lambda=3$. Changing $\Lambda$ affects the results only 


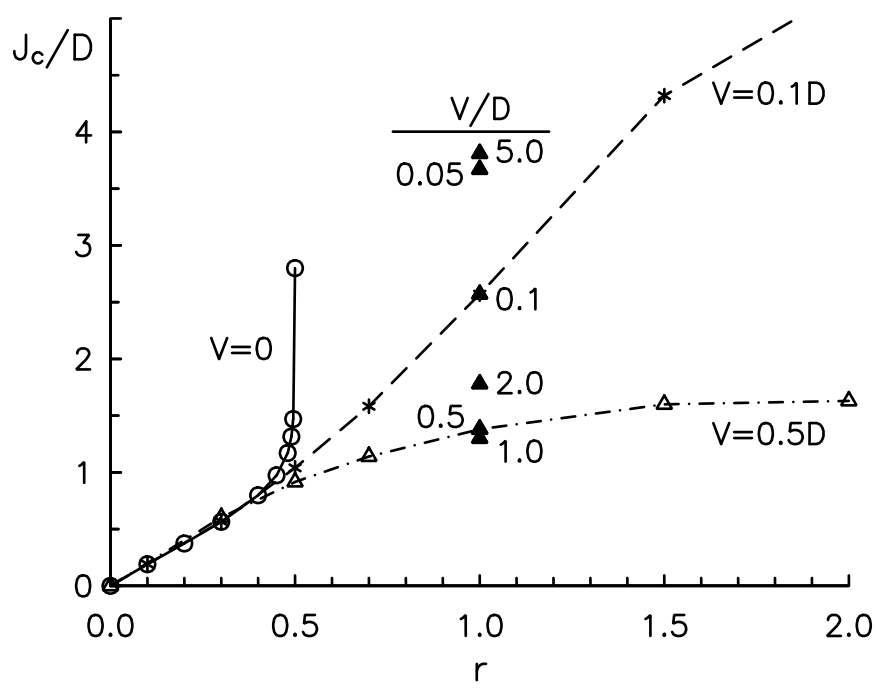

FIG. 1. Critical Kondo coupling $J_{c} / D$ vs. the power $r$ entering Eq. (2), for potential scatterings $V / D=0,0.1$ and 0.5. (The connecting lines are provided as a guide to the eye.) For $r=1$ only, data are shown for additional values of $V$.

through small shifts in the value of $J_{c}$.

Results are presented first for pure power-law densities of states [Eq. (2)], confirming and extending the conclusions drawn above. Consider the particle-hole symmetric case, $V=0$. For $0<r \leq \frac{1}{2}$ there exists a critical coupling $J_{c}(r)$, plotted in Fig. 1. For small $r, J_{c} \approx 2 r D$ (in agreement with Ref. [6]), but the curve $J_{c}(r)$ turns upward and then terminates at $r=\frac{1}{2}$. Far below a crossover temperature, $T_{X} \approx D\left[\left|J-J_{c}\right| / \max \left(J, J_{c}\right)\right]^{1 / r}$, any coupling $J<J_{c}$ yields the same excitation spectrum as $\tilde{H}_{M}^{(0)}$, implying renormalization to zero exchange; whereas values $J>J_{c}$ reproduce the strong-coupling spectrum of $\tilde{H}_{M}^{(1)}$. In the region $r>\frac{1}{2}$, the critical point disappears, so any exchange $0<J<\infty$ renormalizes to zero [9].

Turning to the case $V \neq 0$, one finds as expected that the curve $J_{c}(r, V)$ extends to arbitrary $r$ (see Fig. 1). For $r \gtrsim \frac{1}{2}$, the critical coupling is strongly $V$-dependent: As $|V|$ increases from zero, $J_{c}$ initially drops from infinity. However, once the potential scattering becomes large enough to inhibit hopping of electrons to or from $f_{0}$ states, further increases in $|V|$ serve only to push $J_{c}$ higher. This is illustrated by the $r=1$ data in Fig. 1.

Figure 2 compares impurity susceptibilities for $r=0$, 0.3 and 0.6 . For $r=0, T \chi_{\text {imp }}$ falls monotonically to zero as the temperature decreases - a sign of the complete screening of the impurity moment which occurs for any $J>0$. This should be contrasted with the $r=0.3$ data: For $J=0.3 D, T \chi_{\mathrm{imp}}$ rises at low temperatures towards the weak-coupling value $\frac{1}{4}$; whereas the $J=0.57 D$ curve is almost flat, indicating that $J$ is very close to $J_{c}$. Neither curve is greatly affected by potential scattering (not shown in Fig. 2), and in both cases $S_{\mathrm{imp}} \approx \ln 2$ over the entire temperature range shown. The two $J=0.8 D$

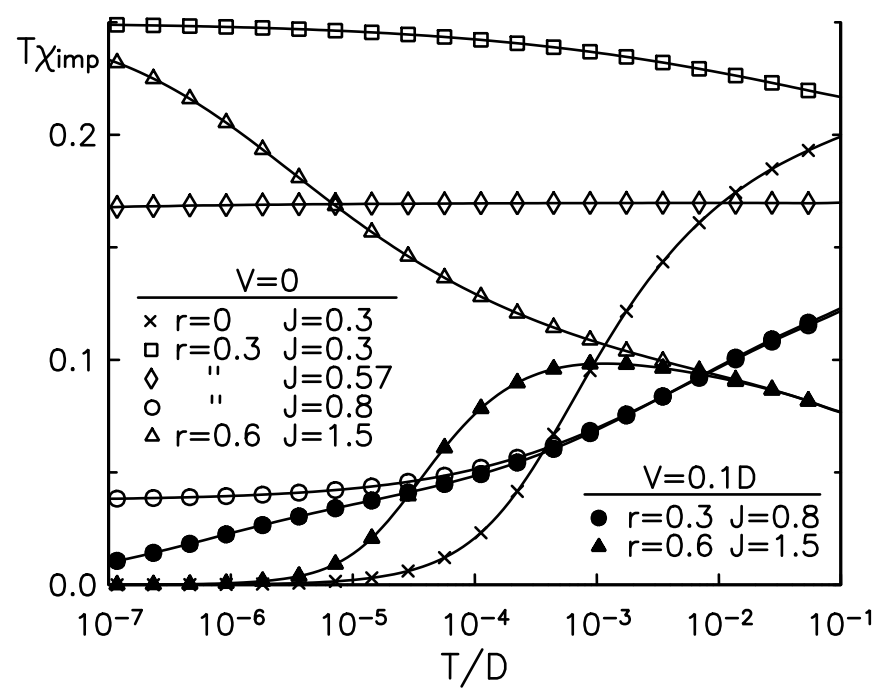

FIG. 2. Impurity susceptibility $T \chi_{\text {imp }}$ vs. $\log (T / D)$, for three power-law densities of states defined in Eq. (2).

curves do exhibit impurity screening at temperatures $T<T_{X} \approx 0.05 D$. For $V=0$, the susceptibility remains Curie-like, reaching a limit $T \chi_{\mathrm{imp}}=0.0377$ very close to the strong-coupling value $r / 8$. The computed entropy, $S_{\mathrm{imp}}=0.416$, is also in good agreement with the predicted value $2 r \ln 2$. Setting $V=0.1 D$ causes a second crossover, around $T_{X}^{\prime}=|V / D|^{1 / r} T_{X} \approx 2 \times 10^{-5} D$, to the particle-hole-asymmetric fixed point, at which $T \chi_{\mathrm{imp}}=0$ and $S_{\mathrm{imp}}=0$. Note, though, that the $V=0$ fixed point still dominates the behavior in the range $T_{X}^{\prime}<T<T_{X}$.

Figure 2 also shows two $r=0.6$ curves. For $V=0$, the impurity remains unscreened at low temperatures, as expected from the vanishing of the intermediate fixed point. If instead $V=0.1 D$, there is a finite critical coupling, $J_{c} \approx 1.3 D$; now an exchange $J=1.5 D$ ensures that the impurity is screened for $T \ll T_{X}^{\prime} \approx 10^{-3} D$.

In order to investigate the sensitivity of the preceding results to the precise form of Eq. (2), the effect of three changes to the density of states will be briefly examined:

(i) In real systems, the power-law variation of $\rho(\epsilon)$ is restricted to an energy range $|\epsilon| \leq \Delta$, with $\rho(\epsilon) \approx \rho(\Delta)$ for $\Delta<|\epsilon| \leq D$. At temperatures $T \gg \Delta$, one finds the standard Kondo physics of metallic systems. For $T \ll \Delta$, however, the impurity "sees" a pure power-law density of states, so the results above still apply, albeit with $J$ replaced by $J_{\text {eff }}(\Delta)>J$. This enhancement makes realization of the Kondo effect more plausible for systems with $r \gtrsim 1$, a range in which the critical couplings would otherwise be unphysically large. Figure 3 illustrates the point: with $\Delta=10^{-3} D$ and $V=0.3 D$, a coupling $J=0.5 D$ fully screens the impurity, for both $r=1$ and 2 .

(ii) Band asymmetry can be introduced by shifting the Fermi level away from the band center, while retaining the power-law variation of $\rho(\epsilon)$ about the new Fermi energy. Although this changes the $\xi_{M-1}$ 's entering Eq. (3), 


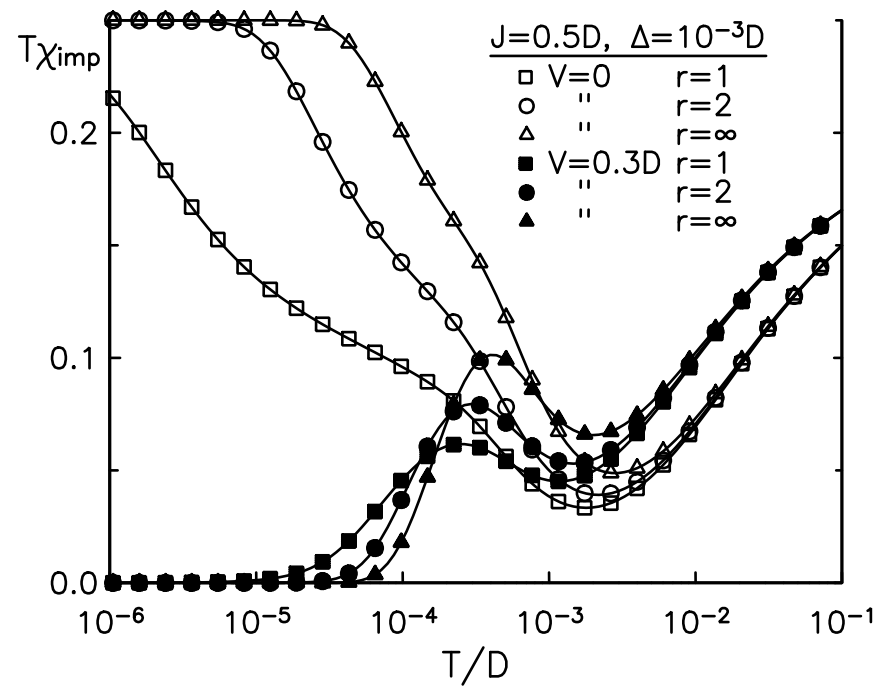

FIG. 3. Impurity susceptibility, $T \chi_{\text {imp }}$ vs. $\log (T / D)$, for restricted power-law densities of states in which $\rho(\epsilon)=\rho_{0}|\Delta / D|^{r}$ for $\Delta<|\epsilon| \leq D$. The $r=\infty$ curves represent an insulator.

and generates nonzero coefficients $\varepsilon_{M}$, the physical effects are little different from those of potential scattering.

(iii) Partial filling of the pseudo-gap may introduce a lower cutoff $\Delta^{\prime}$ on the power-law density of states, with $\rho(\epsilon) \approx \rho\left(\Delta^{\prime}\right)$ for $|\epsilon|<\Delta^{\prime}$. The finite value of $\rho(0)$ must eventually produce a standard, metallic Kondo effect. However, for $r \gtrsim 1$ and most plausible values of $J, \Delta$ and $\Delta^{\prime}$, this occurs at inaccessibly low temperatures.

Discussion: Although large- $N$ studies of gapless systems [6 8] have treated symmetric bands with zero potential scattering, they have found no sign of the Kondo effect disappearing for any power $r$. This discrepancy with Ref. [10] and the present work may stem from the mean-field nature of the large- $N$ method, or from the symmetry-breaking that is implicit, for all $N>2$, in the restriction that the impurity level be singly occupied.

The $r=\infty$ limit of the restricted density of states defined in (i) above describes an insulator with gap $2 \Delta$. The results of this work are entirely consistent with those known for gapped systems. At particle-hole symmetry, an impurity in an insulator retains its moment, no matter how large $J$ is made; away from this symmetry, the spin is screened provided that $J>J_{c} \approx 2 D / \ln (D / \Delta)$ 16. The mapping of an impurity in an $s$-wave BCS superconductor onto Eq. (11) necessarily introduces particle-hole asymmetry, resulting in a finite $J_{c}$ [17]. Figure 3 demonstrates the similarities between the impurity susceptibilities for $r=1,2$ and $\infty$. However, the low-temperature decrease in the impurity resistivity is a signature of gapless systems which has no counterpart in insulators.

This study shows that the behavior of dilute spin impurities in gapless Fermi systems differs qualitatively from that in metals or insulators. In most gapless materials, a sufficiently large exchange coupling, $J>J_{c}$, results in complete screening of the impurity, marked by the vanishing of $T \chi_{\text {imp }}$ and a low-temperature decrease in the impurity resistivity; for $J<J_{c}$, the impurity asymptotically decouples from the band. Systems which lie close to particle-hole symmetry are unlikely to exhibit a Kondo effect because in such cases $J_{c}$ becomes unphysically large. Further work is needed to extend these results to degeneracies $N>2$, to obtain the full temperature dependence of the resistivity, and to treat self-consistently magnetic impurities in unconventional superconductors.

I thank P. Hirschfeld and A. Schiller for many helpful discussions; C. Cassanello, E. Fradkin, and K. Ziegler for informative conversations; L. Oliveira for pointing out the form of Eq. (雨); and T. Costi for drawing my attention to Ref. 10]. This work was supported in part by NSF Grant No. DMR-9316587.

* Affiliated with the National High Magnetic Field Laboratory.

[1] M. Sigrist and K. Ueda, Rev. Mod. Phys. 63, 239 (1991).

[2] Narrow-Gap Semiconductors, Springer Tracts in Modern Physics, Vol. 98, ed. G. Höhler (Springer-Verlag, Berlin, 1983).

[3] B. A. Volkov and O. A. Pankratov, Pis'ma Zh. Eksp. Teor. Fiz. 42, 145 (1985) [JETP Lett. 42, 178 (1985)].

[4] M. P. A Fisher and E. Fradkin, Nucl. Phys. B251 [FS13], 457 (1985); A. W. W. Ludwig, M. P. A. Fisher, R. Shankar, and G. Grinstein, Phys. Rev. B50, 7256 (1994); K. Ziegler, M. H. Hettler, and P. J. Hirschfeld, preprint (cond-mat/9604176).

[5] G. Baskaran, Z. Zou, and P. W. Anderson, Solid State Commun. 63, 973 (1987); A. Ruckenstein, P. J. Hirschfeld, and J. Appel, Phys. Rev. B36, 857 (1987); I. Affleck and J. B. Marston, ibid. 37, 3774 (1988); G. Kotliar, ibid. 37, 3664 (1988).

[6] D. Withoff and E. Fradkin, Phys. Rev. Lett. 64, 1835 (1990).

[7] C. R. Cassanello and E. Fradkin, preprint condmat/9512064).

[8] L. S. Borkowski and P. J. Hirschfeld, Phys. Rev. B46, 9274 (1992); J. Low. Temp. Phys. 96, 185 (1994).

[9] K. Ingersent, to appear in Proceedings of the Conference on Physical Phenomena at High Magnetic Fields II, eds. L. P. Gor'kov et al. (World Scientific, Singapore, 1996).

[10] K. Chen and C. Jayaprakash, J. Phys.: Condens. Matter 7, L491 (1995).

[11] It is assumed throughout that $\int d \epsilon \rho(\epsilon)=1$.

[12] K. G. Wilson, Rev. Mod. Phys. 47, 773, (1975).

[13] The units are such that $k_{B}=g \mu_{B}=1$, where $g$ is the electronic $g$-factor and $\mu_{B}$ is the Bohr magneton.

[14] K. Ingersent (to be published).

[15] W. C. Oliveira and L. N. Oliveira, Phys. Rev. B49, 11986 (1994). 
[16] K. Takegahara, Y. Shimizu, and O. Sakai, J. Phys. Soc. (Japan) 613443 (1992).

[17] K. Satori, H. Shiba, O. Sakai, and Y. Shimizu, J. Phys. Soc. (Japan) 613239 (1992). 